\title{
Effects of Panax ginseng C.A. Meyer extract on the offspring of adult mice with maternal immune activation
}

\author{
HAK-JAE KIM $^{1}$, HANSOL WON ${ }^{1}$, JIYUN IM ${ }^{1}$, HWAYOUNG LEE ${ }^{1}$, JIWOO PARK ${ }^{1}$, \\ SANGHYUN LEE ${ }^{2}$, YOUNG-OCK KIM ${ }^{3}$, HYUNG-KI KIM ${ }^{1}$ and JUN-TACK KWON ${ }^{1}$ \\ ${ }^{1}$ Department of Clinical Pharmacology, College of Medicine, Soonchunhyang University, Cheonan, Chungcheongnam 31151; \\ ${ }^{2}$ Department of Integrative Plant Science, Chung-Ang University, Anseong, Gyeonggi 17546; \\ ${ }^{3}$ Department of Bio-Medical Convergence Development, International St. Mary's Hospital, College of Medicine, \\ Catholic Kwandong University, Incheon 22711, Republic of Korea
}

Received December 21, 2017; Accepted June 12, 2018

DOI: $10.3892 / \mathrm{mmr} .2018 .9417$

\begin{abstract}
To understand maternal immune activation (MIA) during prenatal development, the synthetic double-stranded RNA polyriboinosinic-polyribocytidylic acid [poly(I:C)] has been widely used in animal models to induce behavioral deficits similar to those in schizophrenia and other psychotic disorders. Panax ginseng C.A. Meyer (PG) extract is widely used to treat various kinds of nervous system disorders in Asia particularly China and Korea. The present study aimed to examine the effects of PG extract on MIA offspring using behavioral activity tests and protein expression analyses. Pregnant mice were exposed to poly(I:C) $(5 \mathrm{mg} / \mathrm{kg})$ or vehicle treatment on gestation day 9 , and the resulting MIA offspring were subjected to vehicle or PG $(300 \mathrm{mg} / \mathrm{kg})$ treatment. In the acoustic startle response test, MIA-induced sensorimotor gating deficit was ameliorated by PG. The majority of behavioral parameters measured in the social interaction (non-aggressive or/and aggressive pattern), open field (number/duration of behavior) and forced swimming test (immobility behavior) were significantly altered in the MIA offspring. Western blot and immunohistochemical analyses of the medial prefrontal cortex indicated that the expression levels of certain neurodevelopmental proteins, including dihydropyrimidinase-related 2, LIM and SH3 domain 1, neurofilament medium, and discs large homolog 4 , were decreased in the untreated MIA offspring, whereas PG treatment improved behavioral impairments and increased neurodevelopmental protein expression in MIA offspring. These results suggested
\end{abstract}

Correspondence to: Professor Hak-Jae Kim, Department of Clinical Pharmacology, College of Medicine, Soonchunhyang University, 31 Suncheonhyang 6-gil, Dongnam, Cheonan, Chungcheongnam 31151, Republic of Korea

E-mail: hak3962@sch.ac.kr

Key words: Panax ginseng C.A. Meyer, maternal immune activation, synthetic double-stranded RNA polyriboinosinic-polyribocytidylic acid, schizophrenia that PG may be useful in neurodevelopmental disorder therapy, including psychiatric disorders such as schizophrenia, owing to its antipsychotic effects.

\section{Introduction}

Maternal immune activation (MIA) during pregnancy increases the risk of developing several mental disorders, including autism and schizophrenia (1). Epidemiological studies have provided compelling evidence that perinatal factors such as MIA increase the risk of schizophrenia and associated disorders following prenatal maternal exposure to infection or inflammation $(2,3)$. To replicate such risks in animal models, fetuses can be exposed to MIA by administering viral mimetic double-stranded RNA polyriboinosinic-polyribocytidylic acid [poly(I:C)], a pro-inflammatory cytokine inducer, to pregnant females $(2,4)$. Notably, a broad range of behavioral impairments in rodent offspring due to gestational immune activation have been commonly described in schizophrenia patients, including deficits in prepulse inhibition (PPI) of acoustic startle response (ASR) $(5,6)$ and social interaction $(6)$, as well as brain abnormalities (7). Among the behavioral outcomes investigated following prenatal immune treatment, PPI is considered as an operational measure of sensorimotor gating. Its deficit has been observed in acute/chronic patients with schizophrenia $(8,9)$ and PPI deficit in schizophrenia patients has been well documented $(9,10)$.

Through experimental investigation, the MIA model has provided substantial evidence of psychosis-associated dysfunction in schizophrenia. Psychosis-associated behavioral abnormalities in adult life have etiological significance in the neurodevelopmental perspective of schizophrenia $(5,11,12)$. A previous study demonstrated that treatment with the typical antipsychotic medication clozapine and the atypical antipsychotic haloperidol reverse cognitive impairment in MIA offspring (6). The effects of maternal treatment with certain drugs on MIA offspring have also been observed; clozapine, haloperidol, risperidone, paliperidone and fluoxetine have exhibited protective effects against the emergence of behavioral and structural abnormalities in MIA offspring $(13,14)$. It has been reported that long-term administration of clozapine 
improves cognitive performance in poly(I:C)-induced MIA mice (14). In addition, treatment with risperidone was reported to prevent the majority of neuropathological alterations in a peri-adolescent MIA mouse model (13). Results from our previous study also demonstrated that MIA induces abnormal behavior associated with alterations in neurodevelopmental protein expression level in the offspring (15).

Panax ginseng C.A. Meyer (PG) is one of the most important medicinal plants in Asia (16). In an experimental brain injury model, PG can suppress microglial activation induced by lipopolysaccharide and improve the recovery of motor function after spinal cord injury, thus providing neuroprotection by alleviating post-traumatic inflammatory responses $(16,17)$. Experimental evidence has suggested that it can improve neurotransmission in the brain as an important pharmacological effect of PG $(16,17)$. Ginseng and its constituents are known to have beneficial effects on cognitive performance, memory, and neurodegenerative disease (18). A number of previous studies have focused on the effects of ginseng on central nervous system diseases such as Alzheimer's disease, Parkinson's disease and depression $(19,20)$. However, few studies have investigated the effects of ginseng on schizophrenia. Chen and Hui (21) observed that ginseng has the therapeutic potential to treat various neurological diseases including schizophrenia. Our previous study has also suggested that oral administrated PG can reduces the incidence of schizophrenia-like symptoms in a neurodevelopmental animal model induced by prenatal stress (22).

The aim of the present study was to examine the effects of PG on behavioral abnormalities relevant to schizophrenia in MIA-induced mouse model offspring. It focused on two aspects: First, the manifestation of behavioral symptoms in a MIA model. Second, the influence of PG on expression levels of neurodevelopmental proteins in medial prefrontal cortex (mPFC) of a MIA model. The potential beneficial effects of PG on MIA offspring were examined based on schizophrenia-like behavior and protein levels in mPFC.

\section{Materials and methods}

Preparation of PG extracts. Dried roots of PG were purchased from a local farm in Yunpung (Chungbuk, Republic of Korea). The root specimens were taxonomically identified by an Oriental medicine physician at the National Institute of Horticultural \& Herbal Science, Rural Development Administration (Jeonju, Korea). A voucher specimen (HPR-207) was deposited in the herbarium of Herbal Crop Research Institute (Eumsung, Korea). The majority of traditional Oriental herbal materials are decocted with boiling water. Therefore, water extraction was used in the present study. In addition, ginsenosides are more soluble in water than in organic solvents (17). Briefly, crushed PG materials (200 g) were extracted under reflux with distilled water three times. Water extracts were then combined and lyophilized. The yield was $18.3 \%$ (wt/wt) for PG in a dried state. Extracts were stored at $-20^{\circ} \mathrm{C}$ until use.

Animals. A total of 27 C57BL6/J mice (9 male and 18, female; $18-25 \mathrm{~g}$ ) were purchased from Daehan BioLink Co., Ltd. (Eumseong, Korea). At the age of 8 weeks, the mice were mated in groups of one male with two females. When a vaginal plug was observed during daily checks, female mice were considered pregnant and mice were separated. All animals were housed under standard conditions $\left(21 \pm 3^{\circ} \mathrm{C}\right.$ and $40 \%$ humidity) in a 12-h light/dark cycle and had free access to food and water. All animal procedures were performed in accordance with the Guide for the Care and Use of Laboratory Animals of the National Institutes of Health (23). Experimental procedures were approved by the Institutional Animal Care and Use Committee of Soonchunhyang University (approval no. SCH16-0063; Cheonan).

MIA and drug administration. Pregnant female mouse were single injected on gestation day 9 (GD9) about $200 \mu \mathrm{l}$ of poly(I:C) ( $\mathrm{n}=12$; potassium salt; Sigma-Aldrich; Merck KGaA, Darmstadt, Germany) or saline $(n=6)$ solution via intravenous route at the tail vein under mild physical constraint. Poly(I:C) was dissolved in isotonic $0.9 \% \mathrm{NaCl}$ solution to obtain desired dosage [5 $\mathrm{mg} / \mathrm{kg}$; calculated based on pure form poly(I:C)] (15). All animals were returned to their cages immediately following the injection and left undisturbed until the weaning of the offspring. Powdered PG extracts were dissolved in water and orally administered (300 mg/kg/day) on postnatal day 35 for $\sim 4$ weeks, until postnatal day 65 (24). All groups contained litters of 8 to 15 pups with similar numbers of males and females. Extremely large or small litters were eliminated. The offspring were weaned at 21 days and group-housed. Male offspring $(n=41)$ were selected and used for further experiments. Three experimental groups were tested as adults: i) 'Control' $(n=14)$ group animals were offspring of unstressed mothers; ii) Poly I:C injected group 'MIA' ( $\mathrm{n}=13$ male offspring) animal were offspring of mothers subjected to stress before parturition; and iii) PG group $(n=14$ male offspring) animals were those subjected to injection of Poly I:C followed by PG administration.

Behavioral tests. Behavioral testing was performed when offspring reached 10 weeks of age $(n=10-12 /$ group; $\geq 2$ pups/litter from each group). Only male subjects were included. The number of subjects in each of the three experimental groups was 10 (at least two pups per litter).

PPI. An automated Startle Reflex System (SR-LAB; San Diego Instruments, Inc., San Diego, CA, USA) was used to measure PPI ( $n=10-12$ animals/group). This system consisted of a startle chamber housed in a sound attenuated isolation cabinet equipped with an internal fan and light. A cylindrical transparent acrylic holding apparatus resting on a four-pegged platform within the isolation chamber was used to hold each subject throughout the testing session. Background noise and acoustic stimuli were controlled through the SR-LAB microcomputer and interface assembly. Stimuli were delivered through a speaker that was mounted above the cylindrical holding apparatus. All test chambers were located in a sound attenuated experimental room to minimize external noise, as previously described (25). Background noise was present throughout the test session at $68 \mathrm{~dB}$. Following a $5 \mathrm{~min}$ acclimation period to background noise, trials were presented in a pseudorandom order, including: i) 14 pulse-alone trials, in which a $40 \mathrm{msec}, 120 \mathrm{~dB}$ broadband noise burst was presented; ii) 30 prepulse +30 pulse trials, in which a $20 \mathrm{msec}$ 
broadband noise prepulse at intensities of 3,6 and $12 \mathrm{~dB}$ were applied above the background noise (10 trials at each intensity) preceded the onset of the $120 \mathrm{~dB}$ pulse for $100 \mathrm{msec}$; and iii) 8 non-stimulus trials comprising background noise only. The prepulse intensities used in the protocol did not induce a startle reaction. All trials were presented with an average intertrial interval of $22 \mathrm{sec}$ (range, 15-30 sec). Additionally, four $120 \mathrm{~dB}$ pulse trials were presented at the beginning and the end of the test session, with a series of 60 acoustic stimuli trials. However, they were not used for the calculation of PPI values. Holding chambers were cleaned with $75 \%$ ethanol between test sessions. PPI values were calculated as a percentage score for each prepulse using the following formula: PPI (\%)=100-[(ASR for prepulse+pulse trial)/(ASR for pulse alone trial)] x100 (25). Mean \%PPI was used as an overall measure of the observed treatment for which percentage PPI data were averaged for three prepulses $(14,15)$.

Forced-swim test (FST). FST was performed as described previously $(26,27)$. Mice ( $n=10-12$ animals/group) were gently placed in a large transparent cylinder filled with fresh tap water $\left(25 \pm 2^{\circ} \mathrm{C}\right)$ for $5 \mathrm{~min}$; water was changed between mice. The swimming, climbing and immobility behaviors were recorded with a video camera and by an observer with a stopwatch. The predominant behaviors were counted every 5 sec. Test scores for swimming (horizontal movement throughout the chamber and crossing quadrants), climbing (upward-directed movements up the side of the chamber and jump-ups from the bottom of the chamber) and immobility (no additional activity other than keeping the head above water or tiny whip kicks) behaviors were recorded.

Open field test (OFT). OFT was conducted to assess exploratory activity and reactivity to a novel environment. Mice ( $n=10-12$ animals/group) were removed from their cages and placed individually in the center zone $(17 \times 17 \mathrm{~cm})$ of the open field arena $(50 \times 50 \mathrm{~cm})$ for $20 \mathrm{~min}$; The apparatus was constructed of opaque Polygal. No background noise was provided. The duration in the central zone and the frequency of entry into the central zone were used as indices for anxiety-like behaviors. The box was cleaned with $70 \%$ ethanol between tests to eliminate odors of other mice. The experimenter exited the room and the behavior of the mouse was recorded as described previously (28).

Social interaction test (SIT). SIT was adapted from previous studies ( $\mathrm{n}=10-12$ animals/group) $(29,30)$. Their social interaction partners were the same-sex siblings with approximately the same body weight that resided in the same cage after weaning. The room in which the chamber was located was darkened during testing and the chamber was illuminated with a single $25 \mathrm{~W}$ red light bulb placed $\sim 100 \mathrm{~cm}$ above the base of the chamber. Each session lasted 20 min. Total duration of social play and the number and types of interactions were scored.

Western blot. mPFC tissues ( $\mathrm{n}=5-6$ animals/group; $\sim 0.5 \mathrm{mg} / \mathrm{ml}$ ) were lysed in radioimmunoprecipitation assay buffer containing protease inhibitors (cat. no. EBA-1149; ELPIS-Biotech, Inc., Daejeon, Korea) and centrifuged at
$18,341 \mathrm{x} \mathrm{g}$ for $10 \mathrm{~min}$ at $4^{\circ} \mathrm{C}$. The Bradford assay was used to determine protein concentration. Proteins $(80 \mu \mathrm{g} / \mathrm{lane})$ were subjected to 10 and $12 \%$ SDS-PAGE and subsequently transferred to polyvinylidene difluoride membranes (Merck KGaA, Darmstadt, Germany). Membranes were blocked with $5 \%$ skimmed milk at room temperature for $1 \mathrm{~h}$ and subsequently probed with anti-dihydropyrimidinase-related 2 (Dpys12; 1:1,000; cat. no. 9393; Cell Signaling Technology, Inc., Danvers, MA, USA), anti-LIM and SH3 domain 1 (Lasp1; 1:2,000; cat. no. MAB8991; Merck KGaA), anti-neurofilament medium (Nefm; 1:1,000; cat. no. 2838; Cell Signaling Technology, Inc.), anti-discs large homolog 4 (Dlg4; 1:1,000; cat. no. 3450; Cell Signaling Technology, Inc.) or anti- $\beta$-actin (Actb; 1:1,000; cat. no. sc-81178; Santa Cruz Biotechnology, Inc., Dallas, TX, USA) antibodies overnight at $4^{\circ} \mathrm{C}$. Following washing with $1 \mathrm{x}$ TBST, these membranes were incubated with horseradish peroxidase-conjugated secondary anti-mouse (1:10,000; cat. no. A9044; Sigma-Aldrich; Merck KGaA) or anti-rabbit (1:5,000; cat. no. LF-SA8002; Abfrontier; Adipogen AG, Liestal, Switzerland) for $1 \mathrm{~h}$ at room temperature. Immunoreactive bands were detected using an Enhanced Chemiluminescence kit (ELPIS-Biotech, Inc.). Quantitative measurements of Dpys12, Lasp1, Nefm, Dlg4 and Actb protein expression levels were made using ImageJ software version 1.51k (National Institutes of Health, Bethesda, MD, USA; http://imagej.nih.gov/ij).

Immunohistochemistry. Mice were deeply anesthetized with ethyl ether and perfused with $4 \%$ paraformaldehyde at room temperature for $\geq 30 \mathrm{~min}$. Fixed brains were removed, frozen and sectioned ( $30 \mu \mathrm{m} ; \mathrm{n}=4-5$ animals/group). Frozen mPFC sections were blocked with normal horse serum (cat. no. S-2000; Vector Laboratories, Inc., Burlingame, CA, USA) for $1 \mathrm{~h}$ at room temperature and subsequently incubated with anti-Dpysl2 (1:700; cat. no. HPA002381; Atlas Antibodies AB, Stockholm, Sweden), anti-Nefm (1:100; cat. no. 2838; Cell Signaling Technology, Inc.) or anti-neuronal nuclei (NeuN; 1:100; cat. no. MAB377; Merck KGaA) antibodies overnight at $4^{\circ} \mathrm{C}$. Following incubation, sections were incubated with Cy3-conjugated anti-rabbit (1:500; cat. no. 715-545-151; Jackson ImmunoResearch Laboratories, Inc., West Grove, PA, USA) and anti-mouse (1:800; cat. no. 111-165-003, Jackson ImmunoResearch Laboratories, Inc.) secondary antibodies at room temperature for $1 \mathrm{~h}$. Fluorescent images were captured using a confocal laser-scanning microscope (FV10i; Olympus Corporation, Tokyo, Japan). Images were quantified with ImageJ software (National Institutes of Health, Bethesda, MD, USA) version $1.51 \mathrm{k}$ using a previously described protocol (31).

High-performance liquid chromatography (HPLC) analysis of ginsenosides. Analyses of ginsenosides Rb1, $\mathrm{Rc}, \mathrm{Rd}, \mathrm{Re}$ and $\mathrm{Rf}$ on $10 \mathrm{mg}$ extract were conducted using a reverse-phase HPLC system in room temperature. HPLC chromatograms were recorded with a Waters 1525 Binary HPLC pump equipped with a Waters 2489 UV/VIS detector (Waters, Miami, FL, USA). Chromatographic separation was performed using a SunFire C-18 column $(2.1 \times 50 \mathrm{~mm} ; 5 \mu \mathrm{m}$; Waters) with a mobile phase consisting of water (solvent A) and acetonitrile (solvent B). A gradient elution was used and 
A
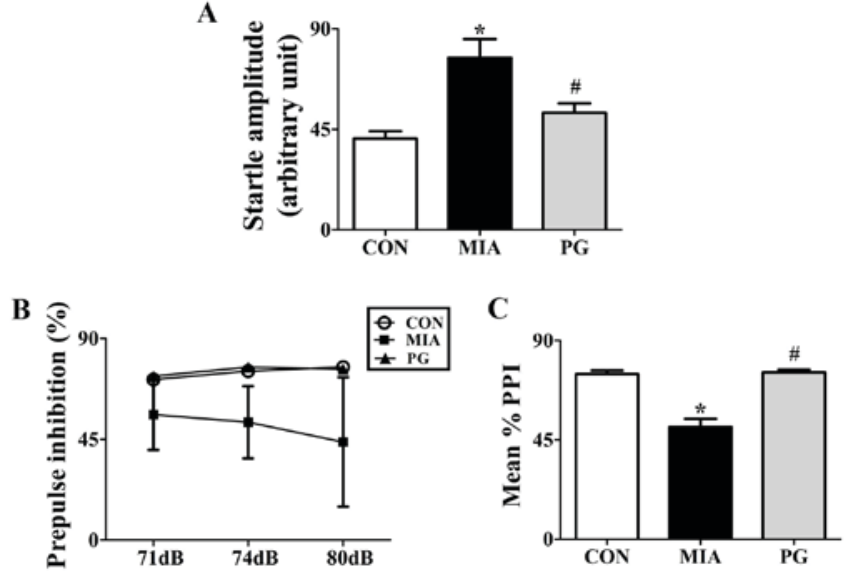

Figure 1. Modulation of PPI and acoustic startle response by PG treatment in MIA mice. (A) Startle amplitude was calculated to $120 \mathrm{~dB}$ of alone stimuli in total performed trials. (B) Inhibition of startle reflex to a $120 \mathrm{~dB}$ stimulus. A stimulus was achieved through prepulses of 71, 74 and $80 \mathrm{~dB}$, corresponding to 3, 6 and $12 \mathrm{~dB}$ above background noise, respectively. (C) PPI is presented as a mean PPI percentage value in startle amplitude as a function of the magnitude of prepulse stimulus. Data are presented as the mean \pm standard error of the mean; " $\mathrm{P}<0.05$ vs. CON; ${ }^{\#} \mathrm{P}<0.05$ vs. MIA. CON, control group; MIA, maternal induced activation group; PG, Panax ginseng-treated MIA group; PPI, prepulse inhibition.

the ratio of these solvents was as follows: 95:5 (A:B) at $0 \mathrm{~min}$, 65:35 (A:B) at $35 \mathrm{~min}$, and 20:80 (A:B) at $40 \mathrm{~min}$. UV detection was set at $204 \mathrm{~nm}$. All injections were conducted three times. The injection volume was $10 \mu \mathrm{l}$ and the flow rate was set at $1.0 \mathrm{ml} / \mathrm{min}$.

Statistical analysis. All data were expressed as the mean \pm standard error of the mean. Data were assessed with one-way analysis of variance (ANOVA) followed by Tukey's post-hoc test. All statistical analyses were performed using SPSS software version 21 (IBM Corp., Armonk, NY, USA). $\mathrm{P}<0.05$ was considered to indicate a statistically significant difference.

\section{Results}

PPI. We tested whether PG treatment to effective in preventing emergence of sensorimotor gating deficiency following MIA during adulthood. To determine the effects of PG on sensorimotor gating function, we measured acoustic startle response (ASR) and performed PPI analysis for Control, MIA-induced PPI disrupted mice and PG treated MIA mice. One-way analysis of variance showed significant effects of PG. It decreased ASR and reversed MIA-induced PPI disruption as shown in Fig. 1. MIA significantly $(\mathrm{P}<0.05)$ increased the acoustic startle amplitude at $120 \mathrm{~dB}$ compared with control group mice (Fig. 1A). PPI values were not significantly reduced $(\mathrm{P}>0.05)$ at 71,74 and $80 \mathrm{~dB}$ following MIA alone compared to those in the control (Fig. 1B). The MIA-induced PPI disruption in $120 \mathrm{~dB}$ pulse stimulus trials (Fig. 1A) was consistently seen across all prepulse levels, leading to significant $(\mathrm{P}<0.05)$ decreases in the mean percent PPI in MIA offspring compared with the control group (Fig. 1C). This indicated that MIA induced sensorimotor gating deficits. Moreover, MIA-induced PPI disruption was significantly $(\mathrm{P}<0.05)$ improved by treatment
Table I. Behavior of CON, MIA and PG mice in the forced swim test.

\begin{tabular}{lrcc}
\hline Behavior & CON & MIA & PG \\
\hline Swimming (n) & $33.86 \pm 1.35$ & $21.57 \pm 1.31^{\mathrm{a}}$ & $33.71 \pm 0.71^{\mathrm{b}}$ \\
Climbing (n) & $18.57 \pm 1.70$ & $10.43 \pm 0.48^{\mathrm{a}}$ & $14.86 \pm 0.96^{\mathrm{b}}$ \\
Immobility (n) & $7.57 \pm 1.39$ & $28.00 \pm 1.00^{\mathrm{a}}$ & $11.43 \pm 0.90^{\mathrm{b}}$ \\
\hline
\end{tabular}

${ }^{\mathrm{a}} \mathrm{P}<0.05$ vs. $\mathrm{CON} ;{ }^{\mathrm{b}} \mathrm{P}<0.05$ vs. MIA; data are presented as the mean \pm standard error of the mean. CON, control group; MIA, maternal induced activation group; n, number of occurrences of the behavior (number calculated every $5 \mathrm{sec}$ ); PG, Panax ginseng-treated MIA group.

with PG. This indicated that PG treatment group animals recovered sensorimotor gating deficits from MIA-induced PPI disruption $(\mathrm{P}<0.05)$.

Forced swim test. There were significant differences in result of FST among Control, MIA and PG treated MIA groups (Table I). Total duration of immobility in MIA offspring was significantly $(\mathrm{P}<0.05)$ increased compared to that in the control. Changed Behavior were significantly $(\mathrm{P}<0.05)$ recovered after $\mathrm{PG}$-treated MIA mice, compared with the untreated MIA group mice $(\mathrm{P}<0.05)$. Each behavior shown that total duration of swimming and climbing decrease in MIA group mice compared with the control and PG treatment groups $(\mathrm{P}<0.05)$.

Open-field test. The number of central entries, line crossings and grooming, and number and duration of cage sniffing behaviors in the MIA group were significantly decreased $(\mathrm{P}<0.05)$ compared with those in the control. However, these scores were reversed $(\mathrm{P}<0.05)$ to normal levels following $\mathrm{PG}$ treatment (Table II). The number and duration of immobility behaviors in the MIA group significantly increased $(\mathrm{P}<0.05)$ compared with the control. These scores were also recovered $(\mathrm{P}<0.05)$ to normal levels following $\mathrm{PG}$ treatment and indicated a recovery from depression behavior (Table II).

SIT. Severe social deficits were induced in the MIA offspring (Table III). The number and duration of cage sniffing and following, in the MIA group were significantly decreased $(\mathrm{P}<0.05)$ compared with the control (Table III). These scores were increased to control levels following treatment with PG. However, aggressive behaviors (for example, fighting and aggressive grooming, including grooming to the neck), excluding biting, during SIT were significantly increased $(\mathrm{P}<0.05)$ in the MIA group compared with the control group (Table III). These scores were decreased to levels observed in the control group following treatment with PG. These results indicated that MIA-induced social interaction impairment was reversed by PG treatment.

Western blot and immunohistochemistry. The expression of neurodevelopmental proteins was altered in the mPFCs of mice prenatally exposed to poly(I:C). To determine whether neurodevelopmental proteins such as Nefm, Lasp1, Dpys12 
Table II. Behavior of CON, MIA and PG mice in the open field test.

\begin{tabular}{|c|c|c|c|}
\hline Behavior & $\mathrm{CON}$ & MIA & PG \\
\hline Central entered (n) & $42.29 \pm 6.11$ & $15.57 \pm 1.31^{\mathrm{a}}$ & $29.57 \pm 4.72^{b}$ \\
\hline Line cross (n) & $22.00 \pm 4.04$ & $5.00 \pm 1.02^{\mathrm{a}}$ & $12.29 \pm 4.86^{\mathrm{b}}$ \\
\hline Run (n) & $0.00 \pm 0.00$ & $0.00 \pm 0.00$ & $0.43 \pm 0.79$ \\
\hline Run (sec) & $0.00 \pm 0.00$ & $0.00 \pm 0.00$ & $0.43 \pm 0.79$ \\
\hline Rear (n) & $96.14 \pm 12.47$ & $74.71 \pm 6.15$ & $69.14 \pm 23.41$ \\
\hline Rear (sec) & $149.57 \pm 9.97$ & $126.86 \pm 8.84$ & $122.86 \pm 35.59$ \\
\hline Grooming (n) & $11.43 \pm 2.86$ & $3.43 \pm 0.57^{\mathrm{a}}$ & $15.71 \pm 3.64^{\mathrm{b}}$ \\
\hline Grooming (sec) & $63.14 \pm 2.73$ & $43.00 \pm 11.01$ & $47.86 \pm 10.57$ \\
\hline Cage sniff (n) & $106.43 \pm 18.76$ & $62.86 \pm 4.97^{\mathrm{a}}$ & $181.71 \pm 30.34^{b}$ \\
\hline Cage sniff (sec) & $221.57 \pm 36.15$ & $123.71 \pm 13.79^{a}$ & $381.86 \pm 77.10^{b}$ \\
\hline Immobile (n) & $3.14 \pm 1.06$ & $25.57 \pm 3.14^{\mathrm{a}}$ & $0.29 \pm 0.76^{b}$ \\
\hline Immobile (sec) & $12.86 \pm 6.61$ & $89.43 \pm 11.72^{\mathrm{a}}$ & $0.43 \pm 1.13^{\mathrm{b}}$ \\
\hline
\end{tabular}

${ }^{\mathrm{a}} \mathrm{P}<0.05$ vs. $\mathrm{CON} ;{ }^{\mathrm{b}} \mathrm{P}<0.05$ vs. MIA; data are presented as the mean \pm standard error of the mean. CON, control group; MIA, maternal induced activation group; n, number of behavior observed; PG, Panax ginseng-treated MIA group.

Table III. Behavior of CON, MIA and PG mice in social interaction test.

\begin{tabular}{lrcr}
\hline Behavior & \multicolumn{1}{c}{ CON } & MIA & PG \\
\hline Sniffing (n) & $50.43 \pm 6.28$ & $21.86 \pm 2.12^{\mathrm{a}}$ & $43.71 \pm 1.96^{\mathrm{b}}$ \\
Sniffing (sec) & $81.29 \pm 8.20$ & $38.43 \pm 5.06^{\mathrm{a}}$ & $80.86 \pm 8.04^{\mathrm{b}}$ \\
Following (n) & $7.86 \pm 1.28$ & $4.57 \pm 0.61^{\mathrm{a}}$ & $11.71 \pm 2.21^{\mathrm{b}}$ \\
Following (sec) & $16.86 \pm 2.34$ & $8.00 \pm 0.90^{\mathrm{a}}$ & $28.00 \pm 6.11^{\mathrm{b}}$ \\
Grooming (n) & $1.71 \pm 0.36$ & $1.00 \pm 0.65$ & $3.86 \pm 0.70^{\mathrm{b}}$ \\
Grooming (sec) & $7.00 \pm 1.72$ & $3.29 \pm 2.15$ & $15.14 \pm 2.63^{\mathrm{b}}$ \\
Fight (n) & $0.29 \pm 0.18$ & $1.29 \pm 0.18^{\mathrm{a}}$ & $0.14 \pm 0.14^{\mathrm{b}}$ \\
Fight (sec) & $0.43 \pm 0.30$ & $4.57 \pm 1.00^{\mathrm{a}}$ & $0.57 \pm 0.57^{\mathrm{b}}$ \\
Aggressive (n) & $0.29 \pm 0.18$ & $1.71 \pm 0.42^{\mathrm{a}}$ & $0.14 \pm 0.14^{\mathrm{b}}$ \\
Aggressive (sec) & $1.14 \pm 0.77$ & $6.29 \pm 2.01^{\mathrm{a}}$ & $0.29 \pm 0.29^{\mathrm{b}}$ \\
Biting (n) & $0.29 \pm 0.18$ & $1.00 \pm 0.49$ & $1.29 \pm 0.52$ \\
Biting (sec) & $0.29 \pm 0.18$ & $2.43 \pm 1.17$ & $1.57 \pm 0.57$ \\
\hline
\end{tabular}

${ }^{\mathrm{a}} \mathrm{P}<0.05$ vs. $\mathrm{CON}$; ${ }^{\mathrm{b}} \mathrm{P}<0.05$ vs. MIA; data are presented as the mean \pm standard error of the mean. CON, control group; MIA, maternal induced activation group; $n$, number of the behavior; PG, Panax ginseng-treated MIA group.

and Dlg4 were downregulated in MIA offspring, western blot analyses were performed. The results revealed that the expression levels of these four proteins in the mPFC were significantly decreased in the MIA group, compared with Control mice (Fig. 2). The MIA-induced reduction in protein expression levels was significantly restored $(\mathrm{P}<0.05)$ following PG treatment. (Fig. 2). Immunohistochemical analyses of mPFC sections revealed that Nefm and Dpysl2 protein expressions were significantly reduced $(\mathrm{P}<0.05)$ in MIA mice compared with mice in the control group (Figs. 3 and 4 , respectively), and treatment with $\mathrm{PG}$ significantly increased Nefm and Dpysl2 expression compared with untreated MIA mice (both $\mathrm{P}<0.05$ ). These results indicated that $\mathrm{PG}$ treatment significantly altered the expression of neurodevelopmental proteins in MIA offspring.

Detection of major ginsenosides. Major ginsenosides, including Rb1, Rc, Rd, Re and Rf of ginseng were detected by HPLC (Fig. 5). Among the major ginsenosides, ginsenoside Rc was the most abundant in ginseng samples. Ginsenoside $\mathrm{Rc}$ is considered the main ginsenoside that contributes to the antioxidant activities of ginseng. Ginsenoside Rc was shown to induce the overexpression of catalase, which inhibited the production of reactive oxygen species in human embryonic kidney 293T (HEK293T) cells (32).

\section{Discussion}

In the present study, it was determined that exposure to MIA resulted in schizophrenia-like behaviors, including impaired sensorimotor gating, decreased PPI and increased startle sensitivity in adult mouse offspring. MIA-induced behavioral impairments were reversed by PG treatment. Western blot and immunohistochemical analyses of the mPFC revealed that several neurodevelopmental proteins were downregulated in MIA offspring compared with the control group, with PG treatment inhibiting this downregulation. To the best of our knowledge, this is the first report to demonstrate that PG treatment reduced behavioral alterations and increased neurodevelopmental protein expression in an MIA-induced animal model of schizophrenia.

A limited number of studies have demonstrated the potential efficacy of the antipsychotics haloperidol and clozapine, or the antidepressant fluoxetine in preventing the onset of psychotic symptoms in a MIA-induced animal model of schizophrenia at GD9 (14). It has been reported that PG exerts similar effects to antipsychotic and antidepressant drugs $(22,33)$.

Ginseng has been demonstrated to have complex pharmacological activities against significant psychiatric symptoms, 

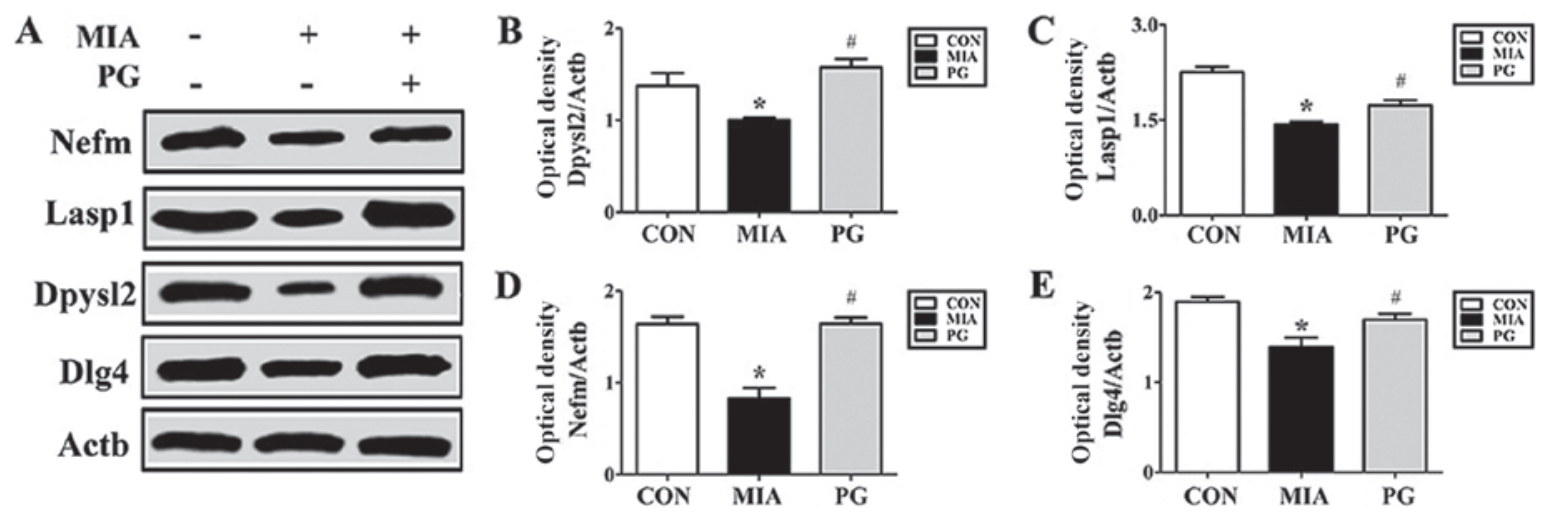

Figure 2. Protein expression levels of Nefm, Lasp1, Dpysl2 and Dlg4 in the medial prefrontal cortex of MIA-induced mice. (A) Protein expression levels were determined by western blot analysis; Actb was used as an internal control. Nefm, Lasp1, Dpys12 and Dlg4 expressions were decreased in the MIA group compared with CON, and treatment with PG inhibited this decrease. (B-E) Quantitative analysis of (B) Dpys12, (C) Lasp1, (D) Nefm and (E) Dlg4 expression. Data are presented as the mean \pm standard error of the mean. ${ }^{*} \mathrm{P}<0.05$ vs. $\mathrm{CON} ;{ }^{*} \mathrm{P}<0.05$ vs. MIA. Actb, $\beta$-actin; CON, control group; Dlg4, discs large MAGUK scaffold protein 4; Dpys12, dihydropyrimidinase like 2; Lasp1, LIM and SH3 protein; MIA, maternal induced activation group; Nefm, neurofilament M; PG, Panax ginseng-treated MIA group.
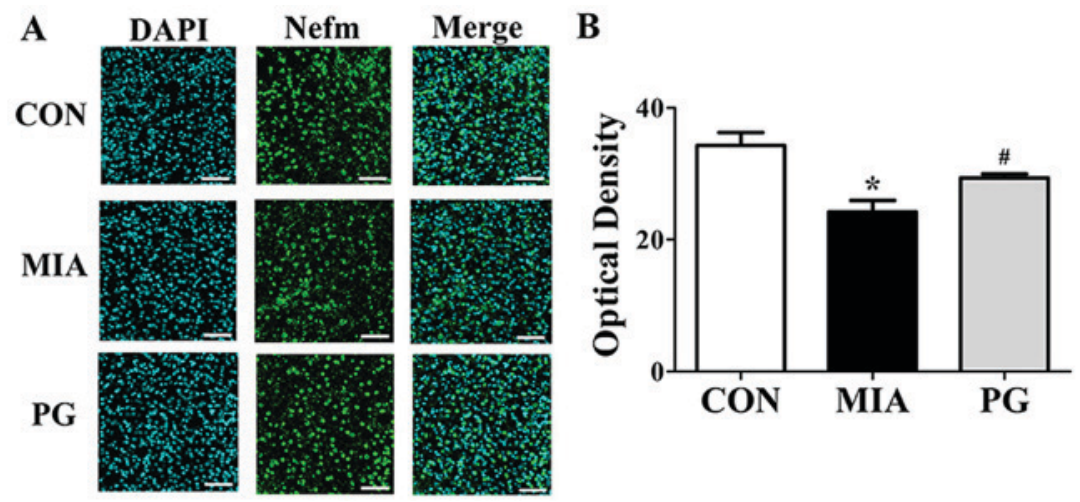

Figure 3. Immunohistochemical analysis of Nefm expression in the medial prefrontal cortex of MIA-induced mice. (A) Confocal microscopy of Nefm immunofluorescent staining (secondary anti-mouse, green; Alexa Fluor 488); nuclei were stained with DAPI (blue). Treatment with PG inhibited this decrease. (B) Quantitative analysis of Nefm expression revealed a significant decrease of Nefm expression in the MIA group compared with CON. Scale bar, $50 \mu \mathrm{m}$. Data are presented as the mean \pm standard error of the mean; ${ }^{*} \mathrm{P}<0.05$ vs. $\mathrm{CON} ;{ }^{*} \mathrm{P}<0.05$ vs. MIA. CON, control group; MIA, maternal induced activation group; Nefm, neurofilament M; PG, Panax ginseng-treated MIA group.
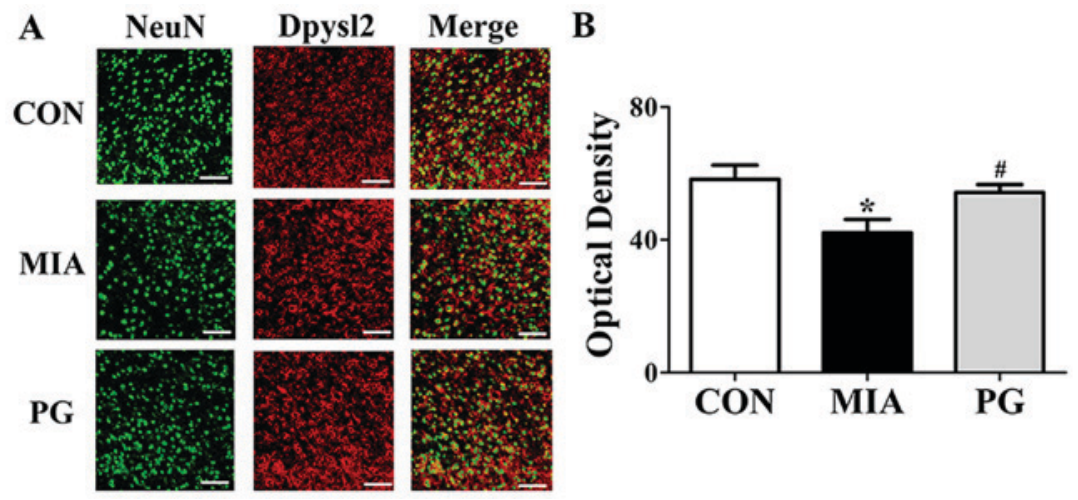

Figure 4. Immunohistochemical analysis of Dpys12 expression in the medial prefrontal cortex of MIA-induced mice. (A) Confocal microscopy of Dpys12 immunofluorescent staining for Dpys12 (secondary anti-rabbit, red; Cy3) with NeuN (secondary anti-mouse, green; Alexa Fluor 488). Scale bar, 50 $\mu \mathrm{m}$. (B) Quantitative analysis of Dpys12 expression revealed a decrease of Dpys12 expression in the MIA group compared with CON, and treatment with PG inhibited this decrease. Data are presented as the mean \pm standard error of the mean; ${ }^{*} \mathrm{P}<0.05$ vs. CON; ${ }^{*} \mathrm{P}<0.05$ vs. MIA. CON, control group; Dpysl2, dihydropyrimidinase like 2; MIA, maternal induced activation group; NeuN, anti-neuronal nuclei; PG, Panax ginseng-treated MIA group.

including sleep disorder, pressure of speech, euphoria, agitation, psychosis, confusion and depression $(34,35)$. Panax vietnamensis has significant and potentially useful psychopharmacological effects: It increases spontaneous locomotor activity, enhances stress endurance, reduces anxiety-like behavior and ameliorates scopolamine-induced memory impairments in mice (36). 


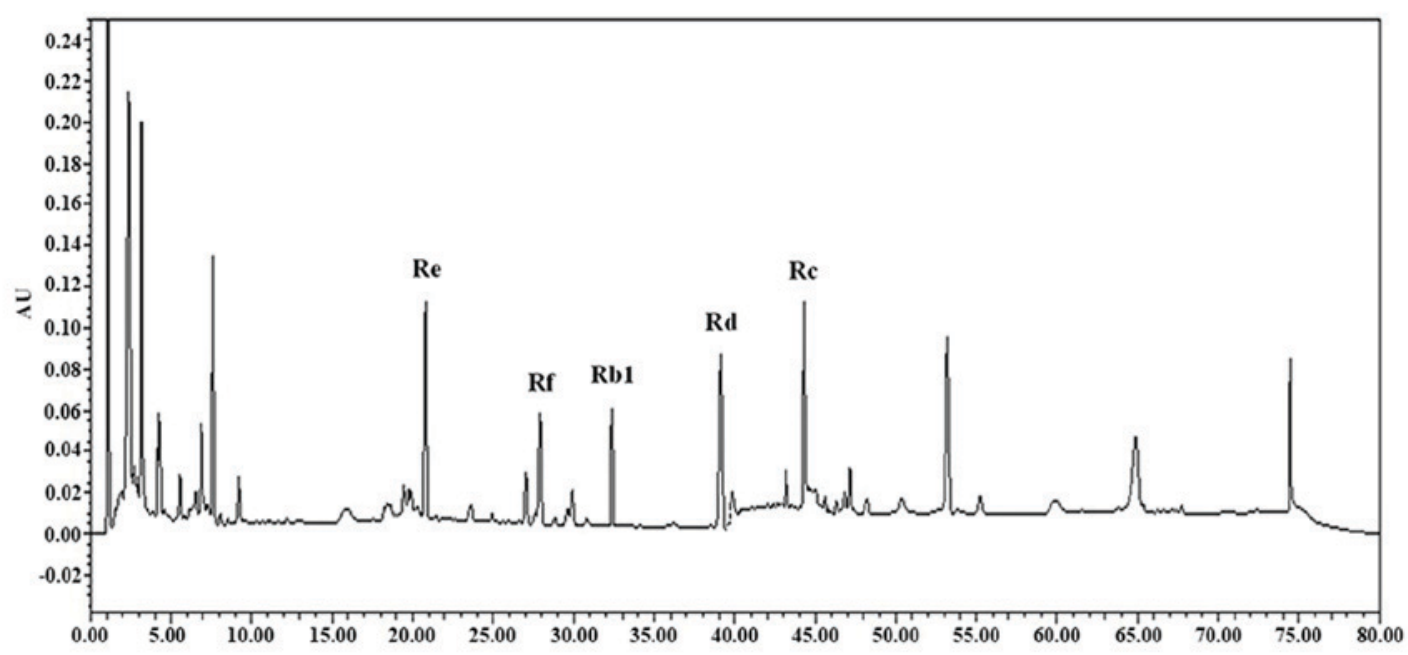

Figure 5. High-performance liquid chromatography of the major ginsenosides, including Rb1, Rc, Rd, Re and Rf.

Crucial components of PG have been reported to possess neuroprotective effects (37) that may decrease psychiatric symptoms (20). In addition, previous studies have revealed that component from ginseng extract may have psychotropic effects and improve agonistic behavior in a mouse model $(38,39)$. Psychotropic actions, including aggressive episodes (offensive sideways posture and attack bite) (38) and maternal aggression (39) in mice are significantly suppressed by ginseng. A previous study reported that Panax quinquefolius is an effective treatment against negative and cognitive symptoms in both acute and chronic animal models of psychosis (40). Our previous study demonstrated that PG extract significantly reduces prenatal stress-induced psychiatric symptoms in an animal model (22). Results from the present study suggested that PG may be considered as a candidate to treat several symptoms of schizophrenia, based on the effects observed in the adult offspring of mice treated with poly(I:C).

A number of previous studies have suggested that impaired PPI of the startle response in patients with schizophrenia, as well as in animal models, may reflect sensorimotor gating disruption $(9,14)$. The present study demonstrated that MIA-induced PPI deficits were markedly reversed by PG. Meyer et al (14) reported the effects of atypical antipsychotic drug administration on PPI deficit in a mouse model of schizophrenia. Moreover, impaired social interaction behavior was observed in MIA mice (14). In particular, MIA may lead to negative and cognitive symptoms of schizophrenia $(41,42)$, including social withdrawal, cognitive dysfunction and anhedonia; in addition, MIA may cause behavioral deficits in adulthood $(41,42)$. The results of the present study supported previous findings that demonstrated that prenatal poly(I:C)-induced MIA in mice is capable of inducing aggressive behaviors (41). In the present study, the MIA-induced decrease in non-aggressive behaviors was inhibited by oral treatment with PG. In addition, certain behavioral patterns observed in the FST and OFT that indicated depressive behaviors were prevented by PG treatment.

The expression levels of Nefm, Lasp1, Dlg4 and Dpys12 proteins were also determined in the present study. The results indicated that application of MIA during crucial periods of fetal brain development resulted in alterations in neurodevelopmental protein expression, including Nefm, Lasp1, Dlg4 and Dpys12. Dpys12, a microtubule-binding protein, has structural and regulatory roles in cytoskeletal dynamics, vesicle trafficking and synaptic transmission in the developing brain $(43,44)$. Furthermore, altered Dpysl2 activity has been associated with schizophrenia in animal models, and Dpysl2 gene polymorphisms are associated with schizophrenia susceptibility in humans $(45,46)$. However, additional research is required to determine the causal link between the effects of PG and reduced schizophrenia-like behaviors. Nevertheless, the present study demonstrated that PG has the potential to be used as a natural alternative remedy for various psychological disorders.

In conclusion, PG and/or its components may be a useful treatment option for schizophrenia. Inhibition of psychotic symptoms may be achieved though the pharmacological effects of PG.

\section{Acknowledgements}

The authors would like to thank Miss Yugyeong Kim (Soonchunhyang University, Cheonan) for technical assistance.

\section{Funding}

The present study was performed with the support of Basic Science Research Program through the National Research Foundation of Korea (NRF) funded by the Ministry of Education (grant no. 2016R1D1A1B03931619).

\section{Availability of data and materials}

The datasets used and/or analyzed during the current study are available from the corresponding author on reasonable request.

\section{Authors' contributions}

H-JK designed and directed the entire project. HW, JI and HL performed the literature searches and analyses. H-KK and JK performed the majority of the statistical analyses, YK, SL 
and JP edited most of the table and figure data, and assisted with statistical analyses. H-JK contributed substantially to the first draft of the manuscript. All authors contributed to, and approved, the final manuscript.

\section{Ethics approval and consent to participate}

All animal procedures were performed in accordance with the Guide for the Care and Use of Laboratory Animals of the National Institutes of Health. Experimental procedures were approved by the Institutional Animal Care and Use Committee of Soonchunhyang University (approval no. SCH16-0063; Cheonan).

\section{Patient consent for publication}

Not applicable.

\section{Competing interests}

The authors declare that they have no competing interests.

\section{References}

1. Brown AS and Patterson PH: Maternal infection and schizophrenia: Implications for prevention. Schizophr Bull 37: 284-290, 2011.

2. Brown AS and Derkits EJ: Prenatal infection and schizophrenia: A review of epidemiologic and ranslational studies. Am J Psychiatry 167: 261-280, 2010.

3. Clarke MC, Harley M and Cannon M: The role of obstetric events in schizophrenia. Schizophr Bull 32: 3-8, 2006.

4. Meyer U, Feldon J and Fatemi SH: In vivo rodent models for the experimental investigation of prenatal immune activation effects in neurodevelopmental brain disorders. Neurosci Biobehav Rev 33: 1061-1079, 2009.

5. Meyer U, Feldon J, Schedlowski M and Yee BK: Towards an immuno-precipitated neurodevelopmental animal model of schizophrenia. Neurosci Biobehav Rev 29: 913-947, 2005.

6. Ozawa K, Hashimoto K, Kishimoto T, Shimizu E, Ishikura H and Iyo M: Immune activation during pregnancy in mice leads to dopaminergic hyperfunction and cognitive impairment in the offspring: A neurodevelopmental animal model of schizophrenia. Biol Psychiatry 59: 546-554, 2006.

7. Meyer U, Feldon J, Schedlowski M and Yee BK: Immunological stress at the maternal-foetal interface: A link between neurodevelopment and adult psychopathology. Brain Behav Immun 20: 378-388, 2006

8. Braff DL, Grillon C and Geyer MA: Gating and habituation of the startle reflex in schizophrenic patients. Arch Gen Psychiatry 49: 206-215, 1992.

9. Braff DL, Geyer MA and Swerdlow NR: Human studies of prepulse inhibition of startle: Normal subjects, patient groups, and pharmacological studies. Psychopharmacology (Berl) 156: 234-258, 2001

10. Ludewig K, Geyer MA and Vollenweider FX: Deficits in prepulse inhibition and habituation in never-medicated, first-episode schizophrenia. Biol Psychiatry 54: 121-128, 2003.

11. Meyer U, Schwendener S, Feldon J and Yee BK: Prenatal and postnatal maternal contributions in the infection model of schizophrenia. Exp Brain Res 173: 243-257, 2006.

12. Meyer U, Nyffeler M, Yee BK, Knuesel I and Feldon J: Adult brain and behavioral pathological markers of prenatal immune challenge during early/middle and late fetal development in mice. Brain Behav Immun 22: 469-486, 2008.

13. Piontkewitz Y, Arad M and Weiner I: Tracing the development of psychosis and its prevention: What can be learned from animal models. Neuropharmacology 62: 1273-1289, 2012.

14. Meyer U, Spoerri E, Yee BK, Schwarz MJ and Feldon J: Evaluating early preventive antipsychotic and antidepressant drug treatment in an infection-based neurodevelopmental mouse model of schizophrenia. Schizophr Bull 36: 607-623, 2010.
15. Won H, Kim YO, Lee H, Im J, Lee S, Cho IH, Lee SW, Park CG, Kim HK, Kwon JT and Kim HJ: Effect of valeriana fauriei extract on the neurodevelopmental proteins expression and behavioral patterns in maternal immune activation animal model. Korean J Med Crop Sci 24: 341-350, 2016.

16. Kim YO, Kim Y, Lee K, Na SW, Hong SP, Valan Arasu M, Yoon YW and Kim J: Panax ginseng improves functional recovery after contusive spinal cord injury by regulating the inflammatory response in rats: An in vivo study. Evid Based Complement Alternat Med 2015: 817096, 2015

17. Park JS, Park EM, Kim DH, Jung K, Jung JS, Lee EJ, Hyun JW, Kang JL and Kim HS: Anti-inflammatory mechanism of ginseng saponins in activated microglia. J Neuroimmunol 209: 40-49, 2009.

18. Rokot NT, Kairupan TS, Cheng KC, Runtuwene J, Kapantow NH, Amitani M, Morinaga A, Amitani H, Asakawa A and Inui A: A role of ginseng and its constituents in the treatment of central nervous system disorders. Evid Based Complement Alternat Med 2016: 2614742, 2016

19. Kim HJ, Kim P and Shin CY: A comprehensive review of the therapeutic and pharmacological effects of ginseng and ginsenosides in central nervous system. J Ginseng Res 37: 8-29, 2013.

20. Lee S and Rhee DK: Effects of ginseng on stress-related depression, anxiety, and the hypothalamic-pituitary-adrenal axis. J Ginseng Res 41: 589-594, 2017.

21. Chen EY and Hui CL: HT1001, a proprietary North American ginseng extract, improves working memory in schizophrenia: A double-blind, placebo-controlled study. Phytother Res 26: 1166-1172, 2012 .

22. National Research Council: Guide for the care and use of laboratory animals. National Academy Press, Washington, DC, 1996.

23. Kim YO, Lee HY, Won H, Nah SS, Lee HY, Kim HK, Kwon JT and Kim HJ: Influence of Panax ginseng on the offspring of adult rats exposed to prenatal stress. Int J Mol Med 35: 103-109, 2015.

24. Nozari M, Shabani M, Farhangi AM, Mazhari S and Atapour N: Sex-specific restoration of MK-801-induced sensorimotor gating deficit by environmental enrichment. Neuroscience 299: 28-34, 2015.

25. Lee HY, Won HS, Im JY, Kim YO, Lee SH, Cho IH, Kim HK, Kwon JT and Kim HJ: Effect of Valeriana fauriei extract on the offspring of adult rats exposed to prenatal stress. Int J Mol Med 38: 251-258, 2016.

26. Renault J and Aubert A: Immunity and emotions: Lipopolysaccharide increases defensive behaviours and potentiates despair in mice. Brain Behav Immun 20: 517-526, 2006.

27. Schroeder M, Sultany T and Weller A: Prenatal stress effects on emotion regulation differ by genotype and sex in prepubertal rats. Dev Psychobiol 55: 176-192, 2013.

28. Lee PR, Brady DL, Shapiro RA, Dorsa DM and Koenig JI: Prenatal stress generates deficits in rat social behavior: Reversal by oxitocin. Brain Res 1156: 152-167, 2007.

29. Shi L, Fatemi SH, Sidwell RW and Patterson PH: Maternal influenza infection causes marked behavioral and pharmacological changes in the offspring. J Neurosci 23: 297-302, 2003.

30. Zhu F, Zheng Y, Liu Y, Zhang X and Zhao J: Minocycline alleviates behavioral deficits and inhibits microglial activation in the offspring of pregnant mice after administration of polyriboinosinic-polyribocytidilic acid. Psychiatry Res 219: 680-686, 2014.

31. Joo J, Lee S, Nah SS, Kim YO, Kim DS, Shim SH, Hwangbo Y, Kim HK, Kwon JT, Kim JW, et al: Lasp1 is down-regulated in NMDA receptor antagonist-treated mice and implicated in human schizophrenia susceptibility. J Psychiatr Res 47: 105-112, 2013.

32. Kim DH, Park CH, Park D, Choi YJ, Park MH, Chung KW, Kim SR, Lee JS and Chung HY: Ginsenoside Rc modulates Akt/FoxO1 pathways and suppresses oxidative stress. Arch Pharm Res 37: 813-820, 2014.

33. Yamada N, Araki H and Yoshimura H: Identification of antidepressant-like ingredients in ginseng root (Panax ginseng C.A. Meyer) using a menopausal depressive-like state in female mice: Participation of 5-HT2A receptors. Psychopharmacology (Berl) 216: 589-599, 2011.

34. Chen XW, Sneed KB, Pan SY, Cao C, Kanwar JR, Chew H and Zhou SF: Herb-drug interactions and mechanistic and clinical considerations. Curr Drug Metab 13: 640-651, 2012.

35. Ernst E: Panax ginseng: An overview of the clinical evidence. J Ginseng Res 34: 259-263, 2010. 
36. Dela Peña IJI, Kim HJ, Botanas CJ, de la Peña JB, Van Le TH, Nguyen MD, Park JH and Cheong JH: The psychopharmacological activities of Vietnamese ginseng in mice: Characterization of its psychomotor, sedative-hypnotic, antistress, anxiolytic, and cognitive effects. J Ginseng Res 41: 201-208, 2017.

37. Smith I, Williamson EM, Putnam S, Farrimond J and Whalley BJ Effects and mechanisms of ginseng and ginsenosides on cognition. Nutr Rev 72: 319-333, 2014.

38. Yoshimura H, Watanabe K and Ogawa N: Psychotropic effects of ginseng saponins on agonistic behavior between resident and intruder mice. Eur J Pharmacol 146: 291-297, 1988.

39. Yoshimura $\mathrm{H}$, Watanabe $\mathrm{K}$ and Ogawa $\mathrm{N}$ : Acute and chronic effect of ginseng saponins on maternal aggression in mice. Eur J Pharmacol 150: 319-324, 1988.

40. Chatterjee M, Singh S, Kumari R, Verma AK and Palit G Evaluation of the antipsychotic potential of Panax quinquefolium in ketamine induced experimental psychosis model in mice. Neurochem Res 37: 759-770, 2012.

41. Bitanihirwe BK, Peleg-Raibstein D, Mouttet F, Feldon J and Meyer U: Late prenatal immune activation in mice leads to behavioral and neurochemical abnormalities relevant to the negative symptoms of schizophrenia. Neuropsychopharmacology 35 : 2462-2478, 2010.
42. Meyer U, Nyffeler M, Engler A, Urwyler A, Schedlowski M, Knuesel I, Yee BK and Feldon J: The time of prenatal immune challenge determines the specificity of inflammation-mediated brain and behavioral pathology. J Neurosci 26: 4752-4762, 2006.

43. Inagaki N, Chihara K, Arimura N, Ménager C, Kawano Y, Matsuo N, Nishimura T, Amano M and Kaibuchi K: CRMP-2 induces axons in cultured hippocampal neurons. Nat Neurosci 4: 781-782, 2001

44. Charrier E, Reibel S, Rogemond V, Aguera M, Thomasset N and Honnorat J: Collapsin response mediator proteins (CRMPs): Involvement in nervous system development and adult neurodegenerative disorders. Mol Neurobiol 28: 51-64, 2003.

45. Lee H, Joo J, Nah SS, Kim JW, Kim HK, Kwon JT, Lee HY, Kim YO and Kim HJ: Changes in Dpysl2 expression are associated with prenatally stressed rat offspring and susceptibility to schizophrenia in humans. Int J Mol Med 35: 1574-1586, 2015

46. Nakata K, Ujike H, Sakai A, Takaki M, Imamura T, Tanaka Y and Kuroda S: The human dihydropyrimidinase-related protein 2 gene on chromosome 8 p21 is associated with paranoid-type schizophrenia. Biol Psychiatry 53: 571-576, 2003.

This work is licensed under a Creative Commons Attribution-NonCommercial-NoDerivatives 4.0 International (CC BY-NC-ND 4.0) License. 